\title{
Plasma N-terminal pro-B-type natriuretic peptide and mortality in type 2 diabetes
}

\author{
L. Tarnow • M.-A. Gall • B. V. Hansen • P. Hovind • \\ H.-H. Parving
}

Received: 12 December 2005 / Accepted: 16 May 2006/ Published online: 26 August 2006

(C) Springer-Verlag 2006

\begin{abstract}
Aims/hypothesis Raised N-terminal pro-B-type natriuretic peptide (NT-proBNP) is associated with a poor cardiac outcome in non-diabetic populations. Elevated NT-proBNP predicts excess morbidity and mortality in diabetic patients with an elevated urinary albumin excretion rate. This study investigated the prognostic value of NT-proBNP in a cohort of type 2 diabetic patients.

Subjects, materials and methods In a prospective observational follow-up study, 315 type 2 diabetic patients with normoalbuminuria $(n=188)$, microalbuminuria $(n=80)$ and macroalbuminuria $(n=47)$ at baseline were followed for a median (range) of $15.5(0.2-17.0)$ years. Plasma NTproBNP concentrations were determined by immunoassay at baseline. Endpoints were overall and cardiovascular mortality.

Results Of the patients, 162 died (51\%), 119 of them (74\%) due to cardiovascular causes. All-cause mortality was increased in patients with NT-proBNP in the second and third tertiles (hazard ratios $[95 \% \mathrm{CI}]$ compared with the first tertile, 1.70 [1.08-2.67] and 5.19 [3.43-7.88], $p<0.001$ ). These associations persisted after adjustment for urinary albumin excretion rate, glomerular filtration rate and conventional cardiovascular risk factors (covariate adjusted hazard ratios 1.46 [0.91-2.33] and 2.54 [1.56-4.14],
\end{abstract}

L. Tarnow $(\bowtie) \cdot$ M.-A. Gall • B. V. Hansen · P. Hovind •

H.-H. Parving

Steno Diabetes Center,

Niels Steensens Vej 2,

DK-2820 Gentofte, Denmark

e-mail: Ltar@steno.dk

H.-H. Parving

Faculty of Health Science, Aarhus University,

Aarhus, Denmark $p<0.001)$. This increased mortality was attributable to more cardiovascular deaths in the second and third NT-proBNP tertile (unadjusted hazard ratios 1.63 [0.96-2.77] and 4.88 [3.01-7.91], $p<0.001$; covariate adjusted 1.37 [0.79-2.37] and 2.26 [1.27-4.02], $p=0.01$ ). When patients with normo-, micro- and macroalbuminuria were analysed separately, NT-proBNP levels above the median $(62 \mathrm{ng} / \mathrm{l})$ were consistently associated with increased overall and cardiovascular mortality in all three groups $(p<0.001)$.

Conclusions/interpretation In patients with type 2 diabetes, elevated circulating NT-proBNP is a strong predictor of the excess overall and cardiovascular mortality, this predictor status being independent of urinary albumin excretion rate and conventional cardiovascular risk factors.

Keywords Albuminuria - Cardiovascular mortality . Mortality $\cdot \mathrm{N}$-terminal pro-B-type natriuretic peptide . Type 2 diabetes · Urinary albumin excretion rate
Abbreviations
BNP B-type natriuretic peptide
IQR interquartile range
NT-proBNP N-terminal pro-B-type natriuretic peptide
ROC receiver operating characteristic

\section{Introduction}

Natriuretic peptides are released from the heart in response to myocardial tension and increased intravascular volume and provide accurate tests for the diagnosis of heart failure compared with echocardiography [1]. B-type natriuretic peptide (BNP) and the N-terminal proBNP fragment (NTproBNP) are both powerful predictors of outcome in patients 
with heart failure at all stages of disease [2]. Recently, data from the Framingham Heart Study identified BNP as a strong predictor of morbidity and mortality in the general population even when BNP levels were below the threshold normally used to diagnose patients with heart failure [3].

Many diabetic patients will succumb to cardiovascular complications. Early identification of vascular risk is the cornerstone of diabetes management and facilitates tailored intervention at an early stage when a beneficial response is more likely to be obtained. Screening for microalbuminuria identifies diabetic patients at increased risk of overt diabetic nephropathy; microalbuminuria is, however, also strongly associated with cardiovascular disease [4, 5]. Furthermore, an elevated plasma NT-proBNP level predicts excess mortality in type 1 diabetic nephropathy [6] and an increased incidence of major cardiovascular events in microalbuminuric type 2 diabetic patients [7].

However, the prognostic importance of NT-proBNP in type 2 diabetic patients in general and the interaction with elevated urinary albumin excretion rate is unknown. Therefore, the present study was performed to assess the effect of plasma NT-proBNP on overall and cardiovascular mortality in a large cohort of type 2 diabetic patients with normo-, microand macroalbuminuria followed prospectively for 15 years.

\section{Subjects, materials and methods}

Study population and protocol

The study population was based on all subjects $(n=363)$ with type 2 diabetes who were $<66$ years of age and attending the Hvidöre Hospital, a tertiary referral centre, during 1987 (baseline [8]). Type 2 diabetes was defined as: (1) diabetes treated by diet alone or by diet combined with oral hypoglycaemic agents; (2) diabetes treated with insulin plus onset of diabetes after the age of 40 years and with patient BMI above normal at time of diagnosis; or (3) diabetes treated with insulin, with patients having a normal BMI and a glucagon-stimulated C-peptide value $\geq 0.60 \mathrm{pmol} / \mathrm{ml}$. We excluded 31 non-Caucasian patients to minimise heterogeneity. In addition four patients who did not hand in baseline urine collections, and 13 in whom NTproBNP could not be determined at baseline were excluded. Clinical congestive heart failure was not an exclusion criteria. The cohort thus consisted of 315 patients.

In a prospective observational study design the patients were followed up until 1 January 2004 or until death $(n=162)$ or emigration $(n=2)$. Data on 9 years of follow-up have been published previously [9]. The study was approved by the local ethics committee, in accordance with the Helsinki Declaration, and all patients gave their informed written consent.
Baseline clinical and laboratory investigations

Patients were interviewed using the WHO cardiovascular questionnaire [10]. Prior major cardiovascular events were defined as a history of stroke and/or myocardial infarction. Smoking was defined as persons smoking one or more cigarettes/cigars/pipes a day; all others were classified as non-smokers. A 12-lead ECG was recorded and subsequently coded independently by two trained observers using Minnesota codes [11]. Known ischaemic heart disease was diagnosed based on the history or an ECG suggestive of myocardial infarction or myocardial ischaemia [5]. Left ventricular hypertrophy was diagnosed by Sokolow-Lyon voltage criteria. Arterial blood pressure was measured twice with a Hawksley random-zero sphygmomanometer and appropriate cuff size following at least a 10-min supine rest. Diabetic retinopathy was assessed in all patients by direct ophthalmoscopy after pupillary dilatation and graded as nil, simplex or proliferative retinopathy. Urinary albumin concentration was measured by RIA [12] from 24-h urine collections. Patients were classified as having normoalbuminuria (albumin excretion $<30 \mathrm{mg} /$ $24 \mathrm{~h}$ ), microalbuminuria (30-299 $\mathrm{mg} / 24 \mathrm{~h}$ ) or macroalbuminuria ( $\geq 300 \mathrm{mg} / 24 \mathrm{~h}$ ) according to established guidelines [13]. Blood was taken in the non-fasting state. $\mathrm{HbA}_{1 \mathrm{c}}$ levels were determined by an isoelectric focusing method [14] (normal range: 4.1-6.1\%), serum creatinine by a kinetic Jaffé method, and serum cholesterol by standard methods. Glomerular filtration rate was estimated by the Modification of Diet in Renal Disease equation [15].

\section{Measurements of NT-proBNP}

After the patients had been at rest for at least $10 \mathrm{~min}$ in the supine position, blood samples for determination of NTproBNP were collected in citrated tubes and centrifuged and plasma stored at $-80^{\circ} \mathrm{C}$ until analysis. Although the stability of NT-proBNP after 15+ years of storage is not known, all samples were treated and stored under the same conditions. Plasma concentrations of NT-proBNP were measured by a sandwich immunoassay on an Elecsys 2010 (Roche Diagnostics, Basel, Switzerland). The intra-assay variation was below $3.0 \%$ and the total CV ranges between 2.2 and $5.8 \%$ in low and high ranges of NT-proBNP. Upper limits of normal NT-proBNP have been determined to be $84 \mathrm{ng} / \mathrm{l}$ in men and $146 \mathrm{ng} / \mathrm{l}$ in women [16].

\section{Follow-up}

All patients were traced through the National Register during summer 2004. If a patient had died before 1 January 2004 the date of death was recorded and information on the cause of death obtained from the death certificate. All death 
certificates were reviewed independently by two observers and the primary cause of death recorded. Additional available information from necropsy reports was included. All deaths were classified as cardiovascular deaths unless an unequivocal non-cardiovascular cause was established [17]. The quality of data does not allow for classification of heart failure as a separate cause of death.

\section{Statistical analysis}

All time-to-death variables were analysed with a log-rank test and displayed on Kaplan-Meier plots according to the presence of nephropathy and/or tertiles of NT-proBNP levels. Cox regression analysis was used to analyse the relationships between baseline normo-, micro- and macroalbuminuria and NT-proBNP levels divided into tertiles on the one hand and mortality/cardiovascular mortality on the other. The relationships were first analysed without adjustment and then after adjustment for the following prespecified baseline cardiovascular risk factors: sex, age, urinary albumin excretion rate, glomerular filtration rate, smoking status, known ischaemic heart disease, left ventricular hypertrophy on ECG, systolic blood pressure and serum cholesterol. Results are described as hazard ratios with $95 \%$ CIs without or with adjustment for other factors that might affect prognosis. A Cox proportional hazards regression model with backwards selection introducing all the above mentioned risk factors was used to evaluate the relative contributions of covariates to mortality, correcting for duration of follow-up.

Analyses of the relationship at baseline between NTproBNP and normo-, micro- or macroalbuminuria were adjusted for sex, age, systolic blood pressure and glomerular filtration rate. For simplicity, these analyses are presented according to median NT-proBNP. Outcome variables that were not normally distributed were logtransformed before analysis and given as medians (interquartile range [IQR]); otherwise means (SD) are presented. Two-tailed $p$ values $\leq 0.05$ were considered statistically significant. Calculations were performed with SPSS version 12.0 (SPSS, Chicago, IL, USA). Areas under the receiver operator characteristic (ROC) curves and their 95\% CIs were estimated and compared using 'roccomb' in STATa 8.0 (StataCorp, College Station, TX, USA).

\section{Results}

After a median follow-up of 15.5 years (range: 0.2-17.0), 162 of the 315 (51\%) type 2 diabetic patients had died, 119 (74\%) due to cardiovascular causes.

The patients were divided into subgroups according to tertiles of NT-proBNP (Table 1). Patients with NT-proBNP in the upper tertiles were older $(p<0.001)$ and had more advanced nephropathy $(p<0.001)$ and retinopathy $(p<0.001)$, lower haemoglobin levels and a higher prevalence of ischaemic heart disease at baseline $(p<0.05)$. NTproBNP levels did not differ between sexes (NS). At baseline, five patients received treatment for heart failure; exclusion of these patients before further analyses did not alter results.

Figure 1 shows Kaplan-Meier curves for overall mortality in patients according to tertiles of NT-proBNP. During follow-up, $31(30 \%)$ patients in the lowest, 47 $(44 \%)$ in the middle and $84(80 \%)$ patients in the upper tertile died $(p<0.001)$. Hazard ratios $(95 \% \mathrm{CI})$ for death in the second and third tertile as compared with the first tertile were $1.70(1.08-2.67)$ and 5.19 (3.43-7.88), respectively $(p<0.001)$. All conventional risk factors, i.e. known ischaemic heart disease, smoking, systolic blood pressure and serum cholesterol were associated with mortality after adjustment for sex and age. Especially, micro- and macroalbuminuria were strongly associated with mortality, with hazard ratios of 1.98 (1.39-2.82) and 3.33 (2.24-4.96), respectively $(p<0.001)$. The NT-proBNP risk estimates were lowered by adjustment for urinary albumin excretion rate, glomerular filtration rate and conventional cardiovascular risk factors; covariate adjusted hazard ratios were 1.46 (0.91-2.33) and $2.54(1.56-4.14)$ in the second and third tertiles, respectively $(p<0.001)$. In particular, the interactions between NT-proBNP and glomerular filtration rate and urinary albumin excretion rate were not significant. In accord, only minor $(<5 \%)$ changes in risk estimates of NTproBNP were observed with the introduction of adjustment for kidney parameters.

The increased mortality was attributable to more cardiovascular deaths in the second and third NT-proBNP tertile (unadjusted hazard ratios 1.63 [0.96-2.77] and 4.88 [3.01-7.91], $p<0.001$; covariate adjusted 1.37 [0.79-2.37] and 2.26 [1.27-4.02], $p=0.01$ [Fig. 2]).

Cox regression analyses including NT-proBNP concentration as a continuous variable revealed an adjusted hazard ratio for all-cause mortality for a tenfold increase in NTproBNP of $2.55(1.72-3.80)(p<0.001)$.

Since a strong association exists between elevated urinary albumin excretion rate and mortality in type 2 diabetes, further analyses of the relationship between NTproBNP and mortality were performed in subgroups of patients with normo-, micro and macroalbuminuria. In comparison with the normoalbuminuric group, plasma NT-proBNP concentrations were elevated in patients with microalbuminuria and macroalbuminuria (median [IQR]): 53 (28-95); 82 (40-222); and 136 (36-524) ng/l, respectively $(p<0.001)$. This difference persisted after adjustment for differences in glomerular filtration rate. In the normoalbuminuric group, 76 patients $(40 \%)$ died, whereas 50 
Table 1 Baseline clinical and laboratory characteristics of 315 type 2 diabetic patients according to tertiles of NT-proBNP

\begin{tabular}{|c|c|c|c|c|}
\hline & First tertile $(n=104)$ & Second tertile $(n=106)$ & Third tertile $(n=105)$ & $p$ \\
\hline Sex (male/female) & $72 / 32$ & $62 / 44$ & $60 / 45$ & 0.14 \\
\hline Age (years) & $50.4(9.1)$ & $53.6(8.5)$ & $58.2(6.8)$ & $<0.001$ \\
\hline Known duration of diabetes (years) & $5(2-11)$ & $5(2-10)$ & $8(4-14)$ & 0.22 \\
\hline Retinopathy (nil/simplex/proliferative) & $82 / 11 / 11$ & $71 / 14 / 21$ & $57 / 12 / 36$ & $<0.001$ \\
\hline Known ischaemic heart disease & 6 & 14 & 40 & $<0.001$ \\
\hline Treated for heart failure & 0 & 0 & 5 & 0.01 \\
\hline Left ventricular hypertrophy on ECG & 3 & 3 & 7 & 0.28 \\
\hline Antihypertensive medication & 29 & 39 & 51 & 0.008 \\
\hline BMI $\left(\mathrm{kg} / \mathrm{m}^{2}\right)$ & $28.9(4.9)$ & $28.6(5.1)$ & $28.4(5.2)$ & 0.80 \\
\hline $\mathrm{HbA}_{1 \mathrm{c}}(\%)$ & $8.3(1.9)$ & $7.8(1.6)$ & $8.2(2.1)$ & 0.20 \\
\hline Nephropathy (normo-/micro-/macroalbuminuria) & $71 / 20 / 13$ & $72 / 25 / 9$ & $45 / 35 / 25$ & $<0.001$ \\
\hline Urinary albumin excretion (mg/24 h) & $17(6-48)$ & $14(6-53)$ & $45(11-216)$ & $<0.001$ \\
\hline Serum creatinine $(\mu \mathrm{mol} / 1)$ & $74(63-84)$ & $73(63-88)$ & $80(69-98)$ & $<0.001$ \\
\hline Estimated glomerular filtration rate $\left.\left(\mathrm{ml} \mathrm{min}-1.73 \mathrm{~m}^{2}\right]^{-1}\right)$ & $100(82-116)$ & $94(78-106)$ & $81(69-93)$ & $<0.001$ \\
\hline Systolic blood pressure (mmHg) & $141(18)$ & $150(21)$ & $160(25)$ & $<0.001$ \\
\hline Diastolic blood pressure (mmHg) & $84(10)$ & $86(11)$ & $86(13)$ & 0.27 \\
\hline Serum cholesterol (mmol/l) & $6.4(1.6)$ & $6.2(1.6)$ & $6.5(1.8)$ & 0.56 \\
\hline Serum HDL-cholesterol (mmol/l) & $1.3(1.3)$ & $1.4(1.7)$ & $1.1(0.4)$ & 0.23 \\
\hline Serum triglycerides $(\mathrm{mmol} / \mathrm{l})$ & $2.1(1.3-3.2)$ & $1.8(1.2-3.0)$ & $1.8(1.2-2.7)$ & 0.31 \\
\hline Haemoglobin (mmol/l) & $10.1(0.8)$ & $9.9(0.8)$ & $9.5(0.9)$ & $<0.001$ \\
\hline Smokers (\%) & 50 & 44 & 47 & 0.17 \\
\hline NT-proBNP (ng/l) & $23(17-32)$ & $62(52-80)$ & $231(139-428)$ & - \\
\hline
\end{tabular}

Data are $n$, means (SD) or medians (IQR)

microalbuminuric (63\%) and 36 macroalbuminuric (77\%) patients died during follow-up $(p<0.001)$. NT-proBNP levels above the median level $(62 \mathrm{ng} / \mathrm{l})$ for the entire population were significantly associated with increased mortality (Fig. 3) and cardiovascular mortality in all three groups of urinary albumin excretion rate.

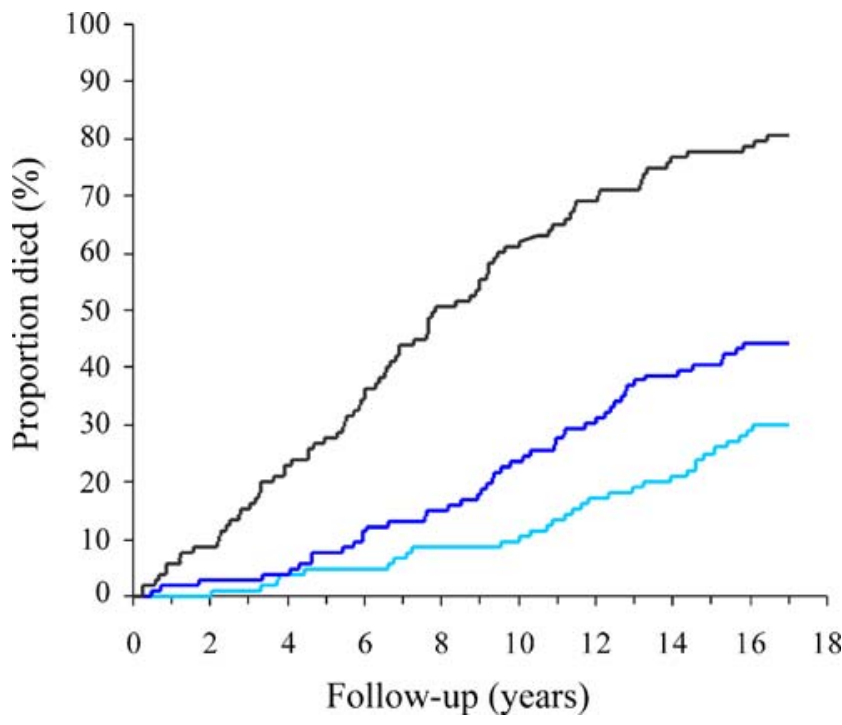

Fig. 1 Kaplan-Meier curves of all-cause mortality in 315 type 2 diabetic patients according to tertiles of NT-proBNP. Black line, upper tertile (NT-proBNP >103 ng/l); dark-blue line, middle tertile; lightblue line, lower tertile (NT-proBNP $<41 \mathrm{ng} / \mathrm{l})$. Log-rank test for overall difference, $p<0.0001$
The overall ( $p=0.69$, Fig. 3 ) and cardiovascular mortality $(p=0.42)$ in patients with macroalbuminuria and a plasma NT-proBNP level below the median were not statistically different from the normoalbuminuric group with NTproBNP above $62 \mathrm{ng} / \mathrm{l}$. A Cox proportional model for mortality is shown in Table 2.

The mean AUCs $(95 \% \mathrm{CI})$ for the ROC curves for NTproBNP and urinary albumin excretion rate were 0.76 $(0.70-0.81)$ and $0.66(0.60-0.72)$, respectively $(p=0.01)$ (Fig. 4). Accordingly, the AUC for traditional cardiovascular risk factors (without NT-proBNP) was $0.82(0.78-0.87)$ and in a model with traditional risk factors and NT-proBNP it was $0.84(0.80-0.89)$ (Fig. 4). At a cut-off value of $62 \mathrm{ng} / \mathrm{l}$, the sensitivity of a single measurement of NT-proBNP for prediction of overall mortality was $69 \%$, whereas the specificity was $70 \%$. The positive and negative predictive values were 71 and $68 \%$, respectively.

\section{Discussion}

Our 15-year prospective observational follow-up study revealed plasma NT-proBNP to be a strong and independent risk marker for all-cause and cardiovascular mortality in type 2 diabetic patients. Importantly, these associations were not only independent of conventional cardiovascular risk factors including known ischaemic heart disease, but also of elevated urinary albumin excretion rate and kidney 


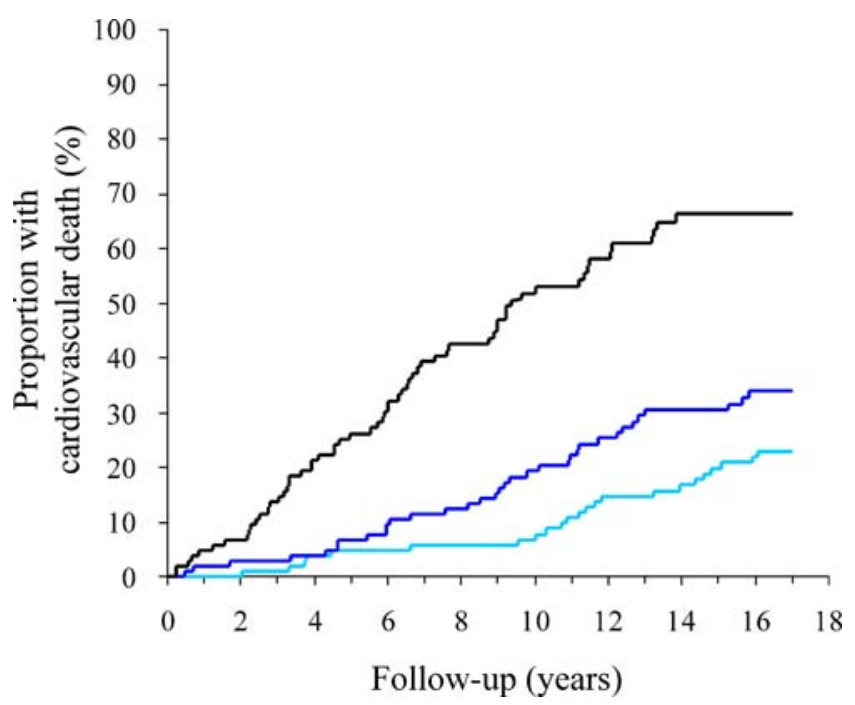

Fig. 2 Kaplan-Meier curves of cardiovascular mortality in 315 type 2 diabetic patients according to tertiles of NT-proBNP. Black line, upper tertile (NT-proBNP >103 ng/l); dark-blue line, middle tertile; lightblue line, lower tertile (NT-proBNP $<41 \mathrm{ng} / \mathrm{l})$. Log-rank test for overall difference, $p<0.0001$

function. Analyses of the relationship between NT-proBNP and outcome in subgroups of patients with normo-, microand macroalbuminuria revealed that a NT-proBNP level above the median value $(62 \mathrm{ng} / \mathrm{l})$ was associated with increased all-cause and cardiovascular mortality in all three groups of urinary albumin excretion rate. Interestingly, type 2 diabetic patients with macroalbuminuria and a NT-proBNP value below the median value had a survival rate comparable with that of normoalbuminuric type 2 diabetic patients with NT-proBNP levels above $62 \mathrm{ng} / \mathrm{l}$. These results thus confirm and extend currently available information on NT-proBNP as a marker of cardiovascular risk in

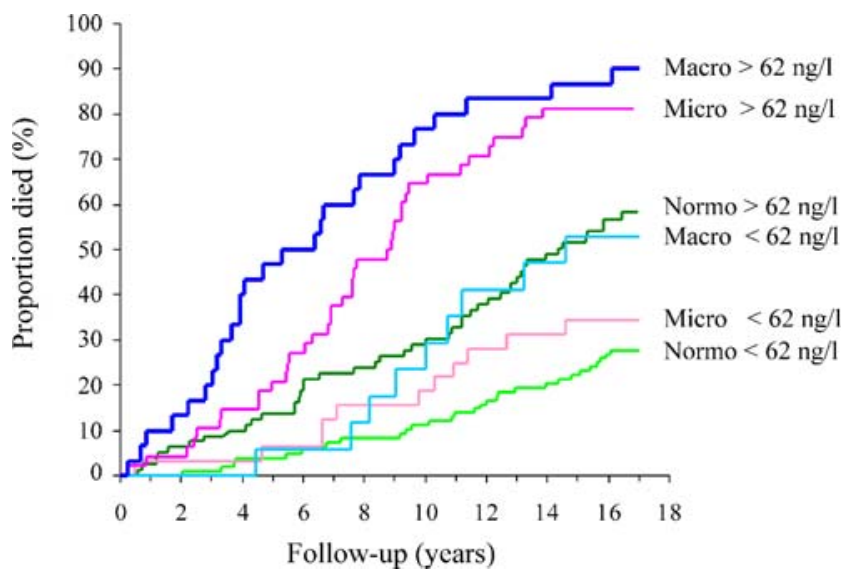

Fig. 3 Kaplan-Meier curves of all-cause mortality in 315 type 2 diabetic patients according to urinary albumin excretion rate and median NT-proBNP for the entire cohort $(62 \mathrm{ng} / \mathrm{l})$. Log-rank test for overall difference, $p<0.0001$ high-risk populations $[6,7]$ to the general type 2 diabetic population.

The interaction between cardiac disease and nephropathy in diabetes has attained increasing recognition [18]. So far, elevated urinary albumin excretion rate has been shown to be the most consistent independent predictor of adverse outcomes in type 1 and type 2 diabetic patients $[8,19]$. In the present study, the predictive value of NT-proBNP on overall mortality was found to be of at least similar magnitude. The kidney also seems to play a role in the clearance of natriuretic peptides, as reflected in the consistent finding of elevated BNP and NT-proBNP levels in diabetic patients with elevated urinary albumin excretion rates in the present and previous studies [6, 20-22]. Our data, however, suggest that only a part of the increased cardiovascular risk in diabetic patients with abnormal urinary albumin excretion rate can be attributed to impaired kidney function, and that NT-proBNP is a risk marker for increased mortality in type 2 diabetic patients with normo-, micro- and macroalbuminuria independently of glomerular filtration rate and level of urinary albumin excretion rate.

The Framingham Heart Study [23] showed that incidence of heart failure for diabetic men was twofold higher than for non-diabetic people and fivefold higher for diabetic vs non-diabetic women. Data from the Breathing Not Properly Multinational Trial furthermore suggest that the prognostic value of BNP in patients who present acutely with dyspnoea is not confounded by the presence of diabetes [24].

Table 2 Cox proportional hazard model of baseline risk factors for all-cause mortality in 315 type 2 diabetic patients followed for 15.5 years

Relative risk
$(95 \% \mathrm{CI})$

\begin{tabular}{lll}
\hline $\begin{array}{l}\text { NT-proBNP } \\
\text { (vs lower tertile) }\end{array}$ & $<0.001$ \\
$\begin{array}{l}\text { Middle tertile } \\
\text { Upper tertile }\end{array}$ & $1.52(0.95-2.41)$ & \\
$\begin{array}{l}\text { Nephropathy } \\
\text { (vs normoalbuminuria) }\end{array}$ & $2.73(1.71-4.35)$ & \\
Microalbuminuria & & $<0.001$ \\
$\quad$ Macroalbuminuria & $1.62(1.12-2.35)$ & \\
Known ischaemic heart disease & $2.66(1.74-4.06)$ & \\
$\quad$ (yes vs no) & $2.13(1.48-3.07)$ & $<0.001$ \\
Age (per year increase) & & \\
$\begin{array}{l}\text { Sex (male vs female) } \\
\text { Smoking (yes vs no) }\end{array}$ & $1.08(1.05-1.11)$ & $<0.001$ \\
LVH on ECG (yes vs no) & $1.64(1.14-2.37)$ & 0.008 \\
Serum cholesterol & $1.41(1.02-1.94)$ & 0.04 \\
(per 1 mmol/l increase) & $2.04(1.01-4.12)$ & 0.05 \\
& $1.09(0.99-1.20)$ & 0.07 \\
\hline
\end{tabular}

Not included in the final model: systolic blood pressure, glomerular filtration rate

$L V H$ Left ventricular hypertrophy 


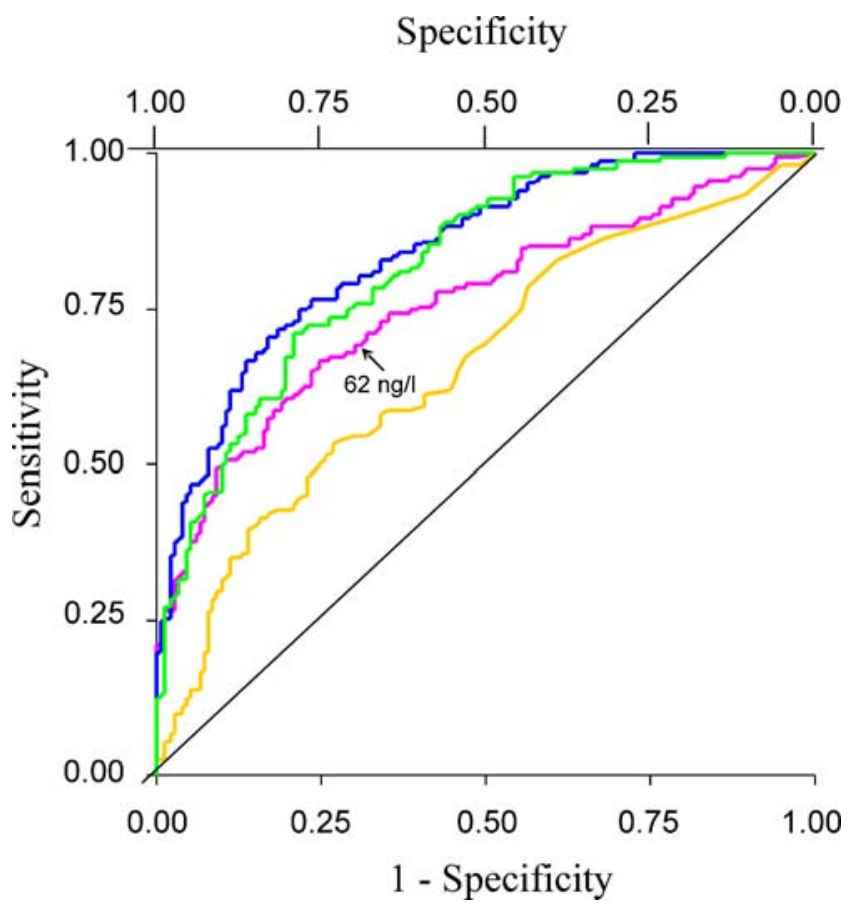

Fig. 4 ROC curves demonstrating the relative ability of NT-proBNP and urinary albumin excretion rate (UAER) to predict overall mortality. The AUC (mean [95\% CI]) for NT-proBNP (pink line) was $0.76(0.70$ $0.81)$ and for UAER (orange line) $0.66(0.60-0.72)(p=0.01)$. The AUC for traditional cardiovascular risk factors (without NT-proBNP; green line ) was $0.82(0.78-0.87)$ and in a model with traditional risk factors and NT-proBNP (blue line) it was $0.84(0.80-0.89)$

While early detection of left ventricular dysfunction enables administration of treatment that can improve survival and well-being $[25,26]$, early ventricular abnormalities may be difficult to diagnose, especially in patients with diabetes, as these patients are often asymptomatic or have non-specific symptoms, and physical examinations are frequently inconclusive. The use of natriuretic peptides in screening programmes for preclinical left ventricular dysfunction in community settings has been debated [27, 28]. However, in high-risk diabetic populations, measurements of BNPs suggest the presence of left ventricular hypertrophy $[29,30]$ and diastolic and systolic dysfunction [31], and predict future cardiovascular morbidity [7] and mortality (present study) [6, 32]. Data on heart function are limited in the present study, since echocardiographies were not performed and the prevalence of ECG-detected left ventricular hypertrophy is low. In a recent study of diabetic patients with diabetic nephropathy, NT-proBNP was found to be closely correlated with left ventricular mass index measured by magnetic resonance scanning [33].

A recent community-based study [3] found a single determination of BNP to provide additional prognostic information and suggested BNP was elevated before the onset of clinically apparent cardiovascular disease in a predominantly $(90 \%)$ non-diabetic population. In accord with a study of male type 2 diabetic veterans [32], and also based on one measurement, our cohort study of type 2 diabetic patients suggests that in clinical practice BNP and NT-proBNP could be useful for the identification of patients with a hitherto unknown high risk of death. These patients might benefit from even more aggressive management of established cardiovascular risk factors and selective referral for further cardiology follow-up. As reported from other studies of asymptomatic patients [3], the relative risk of death and cardiovascular events was more than doubled at NT-proBNP values well below those currently considered diagnostic for heart failure. Based on existing data from diabetic populations and the ROC curve (Fig. 4) a NTproBNP cut-off value of $62 \mathrm{ng} / \mathrm{l}$ is suggested. The clinical impact of programmes concerned with diagnosis, classification and cardioprotective treatments, however, remains to be investigated.

BNP is synthesised predominantly in the ventricular wall in response to increased myocyte stretch following volume expansion, pressure overload or increased cardiac chamber wall stress. Although the present study was not designed to study possible biological mechanisms for increased BNP levels, other studies have demonstrated fluid retention, blood pressure elevation, silent myocardial ischaemia and left ventricular hypertrophy to be frequent in patients with diabetes [34].

The present study adds new information on overall and cardiovascular mortality to data on cardiovascular morbidity from a previous study reporting from a clinical trial [7]. The strengths of our study include its prospective long-term design, the unselected diabetes clinic-based patient population, and the completeness of follow-up. Limitations include its relatively small size, lack of echocardiographic examinations, and changes in treatment guidelines during follow-up, which may have diluted the influence of conventional cardiovascular risk factors such as blood pressure and serum lipids.

In conclusion, in patients with type 2 diabetes, elevated circulating NT-proBNP is a strong predictor of excess overall and cardiovascular mortality, independently of urinary albumin excretion rate and conventional cardiovascular risk factors. The measurement of NT-proBNP adds prognostic information and thus could help to guide management of cardiovascular complications in type 2 diabetic patients.

Acknowledgements We appreciate the laboratory assistance of B. R. Jensen and U. M. Smidt. Roche Diagnostics, Germany provided the kits and measured NT-proBNP. 


\section{References}

1. Doust JA, Glasziou PP, Pietrzak E, Dobson AJ (2004) A systematic review of the diagnostic accuracy of natriuretic peptides for heart failure. Arch Intern Med 164:1978-1984

2. Doust JA, Pietrzak E, Dobson A, Glasziou P (2005) How well does B-type natriuretic peptide predict death and cardiac events in patients with heart failure: systematic review. BMJ 330:625

3. Wang TJ, Larson MG, Levy D et al (2004) Plasma natriuretic peptide levels and the risk of cardiovascular events and death. N Engl J Med 350:655-663

4. Mogensen CE (1984) Microalbuminuria predicts clinical proteinuria and early mortality in maturity onset diabetes. N Engl J Med 310:356-360

5. Gall M-A, Rossing P, Skøtt P et al (1991) Prevalence of microand macroalbuminuria, arterial hypertension, retinopathy and large vessel disease in European Type 2 (non-insulin-dependent) diabetic patients. Diabetologia 34:655-661

6. Tarnow L, Hildebrandt P, Hansen BV, Borch-Johnsen K, Parving $\mathrm{H}-\mathrm{H}$ (2005) Plasma N-terminal pro-brain natriuretic peptide as an independent predictor of mortality in diabetic nephropathy. Diabetologia 48:149-155

7. Gæde P, Hildebrandt P, Hess G, Parving H-H, Pedersen O (2005) Plasma N-terminal pro-brain natriuretic peptide as a major risk marker for cardiovascular disease in patients with type 2 diabetes and microalbuminuria. Diabetologia 48:156-163

8. Gall M-A, Borch-Johnsen K, Hougaard P, Nielsen FS, Parving $\mathrm{H}-\mathrm{H}$ (1995) Albuminuria and poor glycemic control predicts mortality in NIDDM. Diabetes 44:1303-1309

9. Stehouwer CD, Gall MA, Twisk JW, Knudsen E, Emeis JJ, Parving HH (2002) Increased urinary albumin excretion, endothelial dysfunction, and chronic low-grade inflammation in type 2 diabetes: progressive, interrelated, and independently associated with risk of death. Diabetes 51:1157-1165

10. Rose GA (1962) The diagnosis of ischaemic heart pain and intermittent claudication in field surveys. Bull WHO 27:645-658

11. Blackburn H, Keys A, Simonson E, Rautaharju P, Punsar S (1960) The electrocardiogram in population studies: a classification system. Circulation 21:1160-1175

12. Christensen C, Ørskov C (1984) Rapid screening PEG radioimmunoassay for quantification of pathological microalbuminuria. Diab Nephropathy 3:92-94

13. Mogensen CE, Keane WF, Bennett PH et al (1995) Prevention of diabetic renal disease with special reference to microalbuminuria. Lancet 346:1080-1084

14. Mortensen HB (1980) Quantitative determination of hemoglobin A1c by thinlayer isoelectric focusing. J Chromatogr 182:325-333

15. Levey AS, Bosch JP, Lewis JB et al (1999) A more accurate method to estimate glomerular filtration rate from serum creatinine: a new prediction equation. Ann Intern Med 130:461-470

16. Hess G, Runkel S, Zdunek D, Hitzler WE (2005) Reference interval determination for $\mathrm{N}$-terminal-B-type natriuretic peptide (NT-proBNP): A study in blood donors. Clin Chim Acta 360: 187-193

17. Pfeffer MA, Swedberg K, Granger CP et al (2003) Effects of candesartan on mortality and morbidity in patients with chronic heart failure: the CHARM-overall programme. Lancet 362:759766
18. Dikow R, Ritz E (2003) Cardiovascular complications in the diabetic patient with renal disease: an update in 2003. Nephrol Dial Transplant 18:1993-1998

19. Borch-Johnsen K, Andersen PK, Deckert T (1985) The effect of proteinuria on relative mortality in Type 1 (insulin-dependent) diabetes mellitus. Diabetologia 28:590-596

20. Siebenhofer A, Ng LL, Plank J, Berghold A, Hodl R, Pieber TR (2003) Plasma N-terminal pro-brain natriuretic peptide in Type 1 diabetic patients with and without diabetic nephropathy. Diabet Med 20:535-539

21. Yano Y, Katsuki A, Gabazza EC et al (1999) Plasma brain natriuretic peptide levels in normotensive noninsulin-dependent diabetic patients with microalbuminuria. J Clin Endocrinol Metab 84:2353-2356

22. Asakawa H, Fukui T, Tokunaga K, Kawakami F (2002) Plasma brain natriuretic peptide levels in normotensive Type 2 diabetic patients without cardiac disease and macroalbuminuria. J Diabetes Complications 16:209-213

23. Kannel WB, Hjorland M, Castelli WP (1974) Role of diabetes in congestive heart failure: the Framingham study. Am J Cardiol 34:29-34

24. Wu AH, Omland T, Duc P et al (2004) The effect of diabetes on B-type natriuretic peptide concentrations in patients with acute dyspnea: an analysis from the Breathing Not Properly Multinational Study. Diabetes Care 27:2398-2404

25. Devereux RB, Liebson PR, Horan MJ (1987) Recommendations concerning use of echocardiography in hypertension and general population research. Hypertension 9:II97-II104

26. Pfeffer MA, Braunwald E, Moye LA et al (1992) Effect of captopril on mortality and morbidity in patients with left ventricular dysfunction after myocardial infarction. Results of the survival and ventricular enlargement trial. The SAVE Investigators. N Engl J Med 327:669-677

27. Redfield MM, Rodeheffer RJ, Jacobsen SJ, Mahoney DW, Bailey KR, Burnett JC Jr (2004) Plasma brain natriuretic peptide to detect preclinical ventricular systolic or diastolic dysfunction: a community-based study. Circulation 109:3176-3181

28. Ng LL, Loke IW, Davies JE et al (2005) Community screening for left ventricular systolic dysfunction using plasma and urinary natriuretic peptides. J Am Coll Cardiol 45:1043-1050

29. Andersen NH, Poulsen SH, Knudsen ST, Heickendorff L, Mogensen CE (2005) NT-proBNP in normoalbuminuirc patients with type 2 diabetes mellitus. Diab Med 22:188-195

30. Fang ZY, Schull-Meade R, Leano R, Mottram PM, Prins JB, Marwick TH (2005) Screening for heart disease in diabetic subjects. Am Heart J 149:349-354

31. Epshteyn V, Mudalier S, Morrison K et al (2003) Utility of B-type natriuretic peptide (BNP) as a screen for left ventricular dysfunction in patients with diabetes. Diabetes Care 26:2081-2087

32. Bhalla MA, Chiang A, Epshteyn V et al (2004) Prognostic role of B-type natriuretic peptide levels in patients with type 2 diabetes mellitus. J Am Coll Cardiol 44:1047-1052

33. Astrup AS, Kim WY, Tarnow L et al (2005) Cardiac function and mass in asymptomatic type 1 diabetic patients with diabetic nephropathy in relation to NT-proBNP - a cardiovascular magnetic resonance study. Diabetologia 48:8 (Abstract)

34. Parving H-H, Østerby R, Ritz E (2003) Diabetic nephropathy. In: Brenner BM (ed) The Kidney, 7th edn. Saunders, Philadelphia, pp $1777-1818$ 